\title{
The role of the gastrointestinal tract in the pathogenesis of rheumatic diseases
}

\author{
Francesco Ciccia*, Angelo Ferrante, Giuliana Guggino, \\ Giovanni Triolo
}

University of Palermo, Piazza delle Cliniche 2, 90127 Palermo, Italy

\section{Keywords:}

Rheumatoid arthritis

Spondyloarthropathy

Connective tissue diseases

Gastrointestinal tract

Dysbiosis

\begin{abstract}
A B S T R A C T
Dysregulation of the intestinal epithelial barrier in genetically susceptible individuals may lead to both intestinal and extraintestinal autoimmune disorders. There is emerging literature on the role of microbiota changes in the pathogenesis of systemic rheumatic diseases such as rheumatoid arthritis, spondyloarthropathies, and connective tissue diseases. Although the role of the gastrointestinal tract in the pathogenesis of spondyloartropathies is well defined and many studies underline the importance of gastrointestinal inflammation in modulating local and systemic inflammation, the data are inconclusive regarding the effect of dysbiosis on rheumatoid arthritis and connective tissue diseases. This review aims to summarize current data on the role of the gastrointestinal involvement and intestinal microbiota in the pathogenesis of systemic rheumatic disease.
\end{abstract}

() 2016 Elsevier Ltd. All rights reserved.

\section{Introduction}

The gastrointestinal tract regulates the trafficking of macromolecules between the environment and the host through an epithelial barrier mechanism [1]. The intestinal epithelial barrier, together with the gut-associated lymphoid tissue and neuroendocrine network, plays a fundamental role in controlling the equilibrium between tolerance and immunity to non-self-antigens [1]. Dysregulation of this organ in genetically susceptible individuals may lead to both intestinal and extraintestinal

\footnotetext{
* Corresponding author. Tel.: +39 0916552137; Fax: +39 0916552189.

E-mail address: francesco.ciccia@unipa.it (F. Ciccia).
} 
autoimmune disorders [1]. The gastrointestinal tract is colonized by many trillions of microbes that represent the so-called microbiota [2]. These microorganisms are not mere bystanders because they play metabolic, trophic, and protective roles and participate in normal human development and homeostasis [2]. The perturbation of this interaction results in dysbiosis that in turn may influence the susceptibility of the host to many immune-mediated diseases. There is emerging literature on the role of microbiota changes in the pathogenesis of systemic autoimmune disease such as type 1 diabetes, celiac disease, ankylosing spondylitis (AS), and rheumatoid arthritis (RA). This review aims to summarize the current data on the role of the gastrointestinal involvement and intestinal microbiota in the pathogenesis of systemic rheumatic disease.

\section{Rheumatoid arthritis}

RA is a chronic autoimmune inflammatory disorder affecting $0.5-1 \%$ of the population [3]. Although the cause of RA remains elusive, it has been demonstrated that in genetically predisposed subjects carrying the (Human Leukocyte Antigen) HLA-DRB $1 * 04$ cluster, environmental factors such as the oral and gut microbiota may lead to the abnormal activation of the innate and adaptive immunity, which involve cellular and humoral immune responses [4]. This activation leads to the formation of autoantibodies [rheumatoid factors (RF) and antibodies against citrullinated peptides/proteins (ACPA)] and invasion of T cells and B cells into the synovium [4] (Fig. 1). Loss of intestinal integrity and increased intestinal permeability have been demonstrated in RA patients, particularly those receiving non steroid anti-inflammatory drugs (NSAIDs) and those with active joint disease [5-7]. Alteration of intestinal permeability may facilitate antigenic absorption and contribute to the persistence of the disease; however, more studies are required to further clarify this specific issue.

\section{Gastrointestinal microbiota in rheumatoid arthritis patients}

Many studies have asserted the role of gastrointestinal microbiota in the pathogenesis of RA. This is suggested by the evidence that the oral bacteria Porphyromonas gingivalis (PG) is capable of inducing the local production of citrullinated protein [8]. PG is a leading pathogen of chronic periodontitis [9] and produces a unique bacterial enzyme, PG peptidyl-arginine deiminase (PPAD), which has the ability to convert arginine residues in proteins to citrulline [8]. Because protein citrullination alters the protein structure, PPAD may be involved in the alteration of the host signaling network and immune evasion (Fig. 1).

Unlike in the case of the oral microbiome, conflicting data emerge from the studies of the intestinal microbiome in animal models of RA and in humans. Data from murine studies support the role of microbiota in influencing arthritis susceptibility. Liu X et al. [10] performed $16 \mathrm{~S}$ rRNA sequencing to characterize the gut microbiota of DBA1 mice that did or did not develop collagen-induced arthritis and demonstrated marked and significant divergence in the distribution of microbiota after arthritis induction. Mice susceptible to collagen-induced arthritis (CIA) showed enriched operational taxonomic units (OTUs) affiliated with the genus Lactobacillus prior to arthritis onset. With disease development, the abundance of OTUs affiliated with the families Bacteroidaceae, Lachnospiraceae, and S24-7 significantly increased in CIA-susceptible mice. Notably, germ-free mice conventionalized with the microbiota from CIA-susceptible mice showed a higher frequency of arthritis induction than those conventionalized with the microbiota from CIA-resistant mice. In this study, the serum concentration of interleukin (IL)-17 and the proportions of $\mathrm{CD}^{+} \mathrm{T}$ cells and Th17 lymphocytes in the spleen were significantly higher in the susceptible group. Using genetic approaches, Block et al. [11] demonstrated that gut microbiota regulates arthritis through follicular helper $\mathrm{T}$ (Tfh) cells, which are defective in antibiotic-treated mice, but not Th17 cells. In particular, the authors investigated the contribution of Th17 and Tfh cells in the induction of arthritis in a $\mathrm{K} / \mathrm{BxN}$ autoimmune arthritis model that is dependent on segmented filamentous bacteria for the induction of the autoimmune phenotype. The authors particularly studied how microbiota modulates the differentiation of Th17 and Tfh cells. Using genetic approaches, they demonstrated that IL-17 is dispensable for arthritis and that antibiotic treatment inhibits disease in IL-17-deficient animals; this suggests that the gut microbiota regulates arthritis independent of Th17 cells. In contrast, conditional deletion of Bcl6 in T cells blocked Tfh cell 


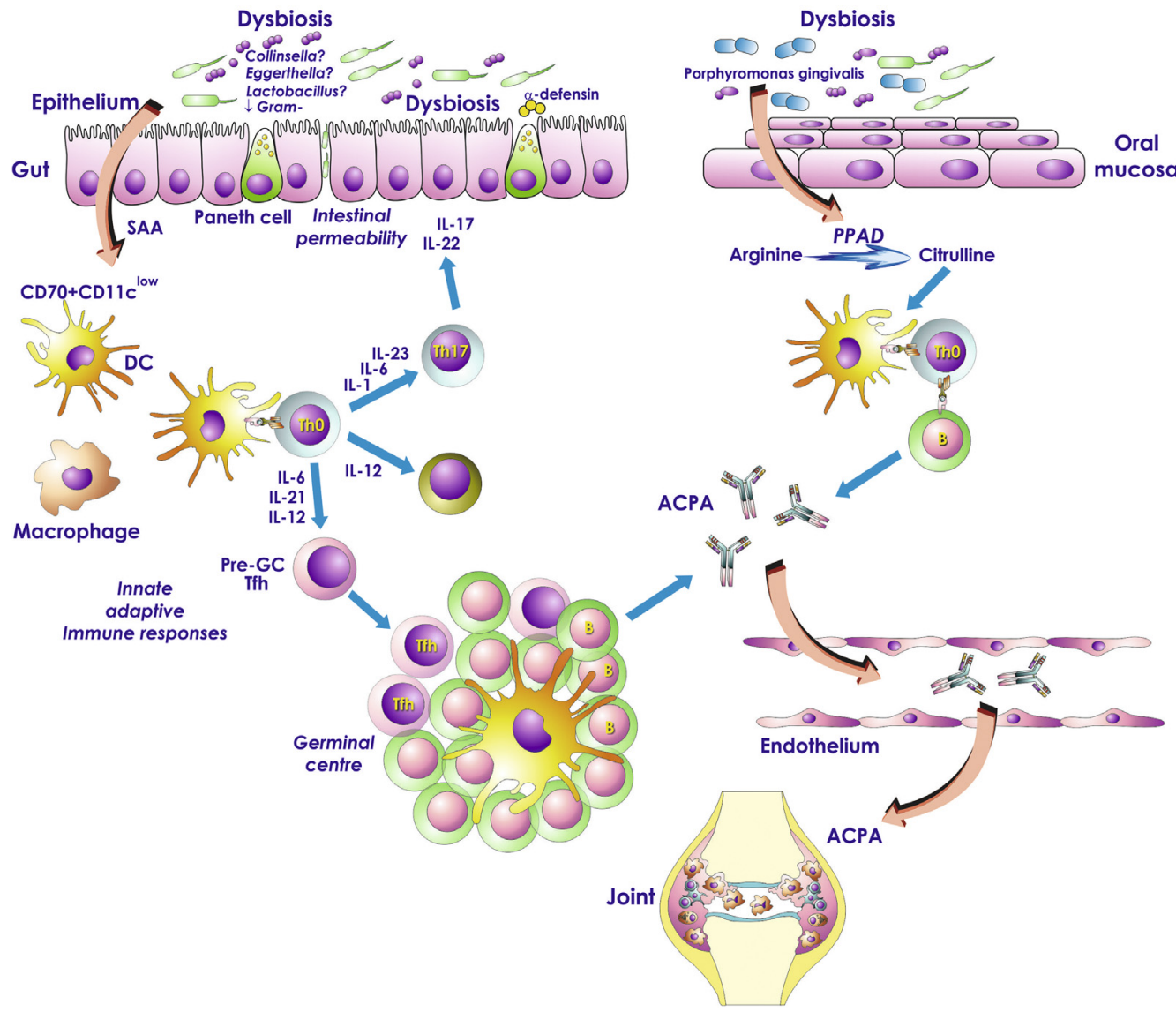

Fig. 1. Dysbiosis in RA: Dysbiosis is present in RA patients and may modulate local and systemic immune responses such as the induction of differentiation of $\mathrm{T}$ helper follicular cells. The role of dysbiosis in modulating other effector $\mathrm{T}$ cells remains to be elucidated. Porphyromonas gingivalis (PG) can induce the local production of citrullinated protein. PG produces a unique bacterial enzyme, P. gingivalis peptidyl-arginine deiminase (PPAD), which has the ability to convert arginine residues in proteins to citrulline. Since that protein citrullination alters protein structure, PPAD may be involved in alteration of the host's signaling network and immune evasion.

differentiation and arthritis development. Furthermore, Tfh cell differentiation was defective in antibiotic-treated mice. Taken together, these findings indicate that gut microbiota regulates arthritis through Tfh but not Th17 cells (Fig. 1).

Data from human studies are more mixed. On the one hand, there is much evidence that supports the association between RA and the intestinal microbiota; bacteria such as Mycoplasma fermentans [12], Escherichia coli [13], and Proteus mirabilis [14] have been reported to initiate the disease. On the other hand, it has been demonstrated that gastrointestinal and urogenital infections are associated with a significantly lowered risk of RA [15] and that patients with RA exhibit decreased gut microbial diversity compared with controls [16]. In particular, it has been proved that expansion of rare taxa such as Actinobacteria along with decrease in abundant taxa occur in patients with RA compared with controls [16]. In this study, three genera, Collinsella, Eggerthella, and Faecalibacterium, were found to be associated with RA; abundance of Collinsella was strongly correlated with the production of the proinflammatory cytokine IL-17A [16]. The role of Collinsella in altering gut permeability and disease severity has been confirmed in experimental arthritis [16]. A more recent study evaluated human fecal Lactobacillus community and its relationship with RA [17]. Samples obtained from RA patients and healthy individuals were analyzed and demonstrated that fecal microbiota of RA patients contained significantly more 
Lactobacillus than that of the control group. In a recently published paper, compositional and functional alterations in RA-associated gut and oral microbiomes were observed, which were partly relieved by disease modifying anti-rheumatic drugs (DMARD) treatment [18]. To delineate the features of the RAassociated gut microbiome, the authors performed a microbiome genome-wide association study identifying 117,219 gene markers that were differentially enriched in RA patients and controls and clustered these genes into metagenomic linkage groups (MLGs) on the basis of their correlated abundance variation among samples. Different from that of the controls, the RA-enriched MLGs formed a large cluster containing strains such as Clostridium asparagiforme, Gordonibacter pamelaeae, Eggerthella lenta, and Lachnospiraceae bacterium and small clusters or single MLGs containing strains such as Bifidobacterium dentium, Lactobacillus sp., and Ruminococcus lactaris. The RA gut was rich in gram-positive bacteria and depleted of gram-negative bacteria, including some Proteobacteria and gram-negative Firmicutes of the Veillonellaceae family. In accordance with the relative depletion of gram-negative bacteria in RA, enrichment of the reductive acetyl-CoA pathway was present in individuals with RA together with modules for converting acetate to methane. Conversely, modules involved in lipopolysaccharide biosynthesis, lipopolysaccharide transport, and secretion systems (type II, type IV, and type VI) were more abundant in samples from healthy controls. Furthermore, several correlations with prognostic or diagnostic factors were found. For instance, the abundance of RA-enriched MLGs such as $C$. asparagiforme and Bacteroides sp. was positively correlated with titers of immunoglobulin A (IgA) and unclassified Lactobacillus sp. (most likely L. salivarius) was positively correlated with titers of the major serum immunoglobulin, IgG. A positive correlation between Enterococcus faecalis and platelet count was also detected. Bacteroides plebeius, Streptococcus australis, Veillonella sp., and Haemophilus parainfluenzae were found to be negatively correlated with titers of the RA-specific autoantibodies ACPA and rheumatoid factor (RF). Furthermore, gut MLGs fit the clinical indices, suggesting that the gut MLGs to some extent could reflect the clinical variations among these subjects. Interestingly, molecular mimicry between RA-associated antigens such as Collagen XI and HLA-DRB $1{ }^{*} 0401$ and gut microbial genes from Clostridium, Eggerthella, Bacteroides, and Citrobacter was also observed. The above-mentioned studies were performed on patients with established RA. However, early identification of RA is a crucial step for controlling the progression of the disease, and studying the bacterial composition in early stages of disease may provide interesting information on its pathogenesis. Fecal microbiota in patients with early RA and patients with Fibromyalgia has been recently studied and compared [19]. In this study, RA patients showed significantly less bifidobacteria and bacteria from the Bacteroides-Porphyromonas-Prevotella group, Bacteroides fragilis subgroup, and Eubacterium rectale-Clostridium coccoides group.

Probiotics are microorganisms that can normalize gut microbiota and are potentially useful to alleviate RA symptoms. Effects of probiotic supplementation on disease activity and inflammatory cytokines in patients with RA was recently studied by Vaghef-Mehrabany [20]. In this study, the disease activity score was significantly decreased by the probiotic supplementation. Tumor necrosis factor (TNF)- $\alpha$, IL-6, and IL-12 were also significantly decreased in the probiotic group, whereas serum level of the regulatory cytokine IL-10 was increased by the supplementation.

\section{Spondyloarthritis}

Spondyloarthritis (SpA) is a generic term used for indicating inflammatory diseases that involve both the joints and entheses (the sites where the ligaments and tendons attach to the bones) [21]. The prototype of these diseases is AS. Other diseases include reactive arthritis, psoriatic arthritis (PsA), and arthritis associated with inflammatory bowel disease. Involvement of the gastrointestinal tract in the pathogenesis of SpA is strongly suggested because subclinical gut inflammation is present in up to $60 \%$ of patients with SpA [22]. SpA-associated gut inflammation has been demonstrated to be an important prognostic factor in SpA because it is associated with more extensive bone marrow edema of the sacroiliac joints and a higher risk of progression to AS [23] and of developing Crohn's disease (CD) [24].

\section{Histologic changes in the gut of SpA patients}

Historically, gut inflammation in SpA patients has been classified into two categories: acute inflammation, which resembles a self-limiting bacterial enterocolitis, and chronic inflammation, which 
displays altered intestinal architecture with varying degrees of infiltration of inflammatory mononuclear cells that are sometimes aggregated in lymphoid follicles, resembling the ileo-colitis observed in CD [22]. More recently, other relevant intestinal alterations have been demonstrated in AS gut: (i) goblet cell hyperplasia and increased mucin production [25], (ii) activation of paneth cells (PC) [26] and hyperplasia of PC [27], particularly in PsA patients, (iii) detachment of epithelial cells from the basal membrane [28], and (iv) occurrence of vasculitic lesions [28] that are mainly represented by intense hemorrhagic extravasation of the lamina propria. Interestingly, similar tissue damages have been described as a consequence of the response of epithelial cells to bacterial toxins [29]. The relative contribution of the genetic factors involved in SpA pathogenesis in influencing these histologic changes cannot be excluded and requires further study. The diagnosis of intestinal inflammation in SpA is essentially made using endoscopy as reliable biomarkers are lacking. Recently, however, elevated serum calprotectin and C-reactive protein (CRP) were demonstrated to be independently associated with microscopic bowel inflammation [30]. During intestinal inflammation, fecal calprotectin is elevated because of the migration of neutrophils to the inflamed intestinal mucosa [31]. In this study, the authors suggested a screening approach where initially serum calprotectin, CRP, and, if necessary, fecal calprotectin are assessed. The model using this scenario provided an area of $74.4 \%$ under the Receiver Operating Characteristic (ROC) curve for the detection of bowel inflammation [30].

\section{Immune alterations in the gut of SpA patients}

In addition to the presence of histologic alterations, the intestine of SpA patients is characterized by the abnormal activation of the innate and adaptive immune system and specific immunologic signatures [32] (Fig. 2). Subclinical gut inflammation in patients with PsA seems to be characterized by the overexpression of IL-9 and presence of fully developed Th9, Th17, and Th22 responses [27]. In particular, in PsA, gut PC express a specific IL-9 receptor, and stimulation of isolated epithelial cells with IL-9 up-regulates the expression of the antimicrobial peptide $\alpha$-defensin 5 and cytokines, such as IL-23 and IL-9, which suggests the possibility of a functional autocrine loop that involves IL-9/IL-9R [27]. Th9 cells also increase in the peripheral blood and synovial tissues in PsA and express $\alpha 4 \beta 7+$, indicating the intestinal origin of these cells [27]. Conversely, in patients with AS, IL-23 over-expression dominates and is mainly detected in the presence of PC without a clearly defined Th17, Th1, and/or Th9 polarization and Treg expansion [33,34].

The intestinal tract immune system is equipped with highly complex structures including the tissue-specific organized lymphoid structures, the Peyer's patches (PPs), cryptopatches, and isolated lymphoid follicles (ILFs) [35]. ILFs are induced postnatally by commensal microbiota and require the presence of specific lymphoid structures called cryptopatches that contain lymphoid tissue inducer (LTi) cells [36]. The recruitment and retention of lymphocytes into the cryptopatches requires IL-7 signaling on IL-7R-bearing LTi cells (a subtype of ILC3) [36]. This signal induces the expression of lymphotoxin $\alpha 1 \beta 2$ (LT- $\alpha 1 \beta 2$ ) that in turn acts on resident lymphoid tissue organizer (LTo) cells [36]. Cryptopatches are virtually absent in the ileum of normal subjects [37], suggesting that bacterial stimulation is necessary for them to develop in physiological conditions. Conversely, in the ileum of AS patients, increased expression of IL-7, mainly in the presence of PC, is associated with the presence of aggregates of LTi cells (CD3-c-kit+/IL-7R+) that resemble murine cryptopatches [37] (Fig. 2). In addition to their role in modulating the formation of cryptopatches, AS intestinal epithelial cells induce the differentiation of type III innate lymphoid cells (ILCs) [37]. ILCs are heterogeneous populations of lymphoid cells that do not display rearrangement of antigen receptors [38]. ILCs are distinguished into three groups: IFN- $\gamma$-producing ILC1 cells, T helper type 2 cytokine-producing ILC2 cells, and IL-17- and IL-22-producing ILC3 cells that are essentially associated with the gastrointestinal mucosa [38]. In AS patients, ILC3 are expanded in the gut and produce high levels of IL-17 and IL-22 [37]. It seems reasonable that, under specific tissue-dependent factors, different subsets of ILC3s producing different cytokines may act promoting different innate immune responses. In this scenario, ILC3s-producing IL22 might be involved in tissue protective responses such as the induction of goblet cell hyperplasia and increased mucin production, observed in AS gut. Conversely, IL-17- and IL-17/IL-22-producing cells might be involved in eliciting pro-inflammatory actions. Interestingly, ILC3s are expanded also in the peripheral blood, synovial fluids, and bone marrow of AS patients. These extra-mucosal ILC3 express 


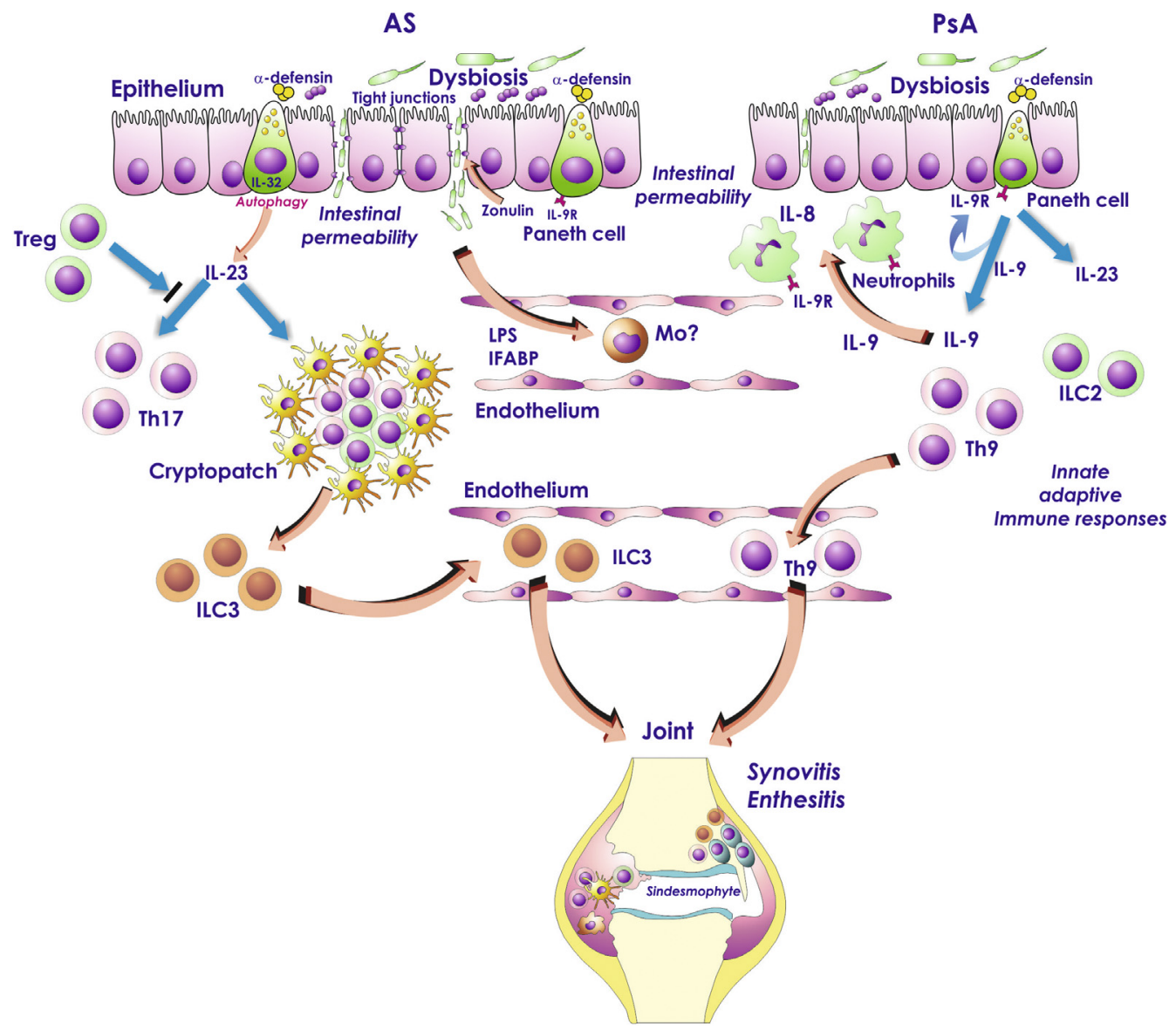

Fig. 2. Subclinical gut inflammation in SpA patients is characterized by the presence of dysbiosis that seems to be responsible for the loss of epithelial integrity and the systemic translocation of bacterial products. Circulating gut-derived bacterial products might influence the function of circulating monocytes and antigen presenting cells. IL-23 immunologically characterizes AS-associated gut inflammation and it seems to play an important role in SpA-associated gut inflammation by inducing the formation of cryptopatches and the expansion of ILC3 that are able to re-circulate in extra-intestinal sites. Subclinical gut inflammation in PsA is immunologically characterized by IL-9 over-expression and Th9 polarization. In PsA, gut-derived Th9 cells may re-circulate from the gut to extraintestinal sites.

the intestinal homing integrin $\alpha 4 \beta 7$ [37]. The $\alpha 4 \beta 7$ ligand, MAdCAM1, has been demonstrated to be highly expressed in the HEVs of the gut and in the inflamed bone marrow (BM) of AS [37], suggesting that a recirculation of ILC3 between the gut and the BM occurs (Fig. 2). The importance of the $\alpha 4 \beta 7 /$ MAdCAM1 axis in modulating tissue inflammation in AS, may be indirectly emphasized by the clinical and radiographic efficacy of $\alpha 4 \beta 7$ block with Natalizumab in AS patients [39]. In the light of these facts, modulation of ILC3 trafficking might be useful as a therapeutic strategy in AS. It is interesting to note that anti-TNF therapy, a well established anti-inflammatory therapy in AS, results in systemic and intestinal reduction of ILC3 and in strong reduction of intestinal expression of MAdCAM1 [37]. Mucosal-associated invariant T (MAIT) cells-a novel innate-like T-cell population, recognizing a highly conserved antigen derived from the microbial riboflavin synthesis pathway [40] - have been recently implicated in the pathogenesis of AS. Gracey E et al. [41] recently demonstrated that MAIT cells are reduced in frequency in the blood of patients with AS compared with HCs. AS patients, however, have an elevated frequency of circulating and synovial IL-17A+ MAIT cells, and IL-17 elevation in AS MAIT cells seems to be dependent on priming with IL-7, but not IL-23 or antigen stimulation. 
Intestinal inflammation is strongly associated with the mucosal microbiota profile of SpA patients as demonstrated in the recent study from Tito et al. [42]. In this study, the bacterial community composition of inflamed biopsies clustered separate from that of non-inflamed biopsies. Interestingly, the authors demonstrated a positive correlation between the abundance of the genus Dialister and the Ankylosing Spondylitis Disease Activity Score (ASDAS). This finding was further supported by the low frequency of Dialister observed in non-inflamed SpA samples and healthy controls. A recent study evaluated the community composition of gut microbiota in patients with PsA, patients with isolated psoriasis of the skin, and healthy control subjects [43]. Samples were further assessed for the presence and levels of fecal and serum secretory $\operatorname{IgA}(\operatorname{sig} A)$, proinflammatory proteins, and fatty acids. The gut microbiota observed in patients with PsA and patients with skin psoriasis was characterized by the reduced presence of several taxa. Samples from both patient groups showed a relative decrease in the abundance of Coprococcus species, while samples from PsA patients were also characterized by a significant reduction in Akkermansia, Ruminococcus, and Pseudobutyrivibrio. Supernatants of fecal samples from PsA patients revealed an increase in sIgA levels and decrease in receptor activator of nuclear factor kappa-B ligand (RANKL) levels. Analysis of fatty acids revealed low fecal quantities of hexanoate and heptanoate in both patients with PsA and patients with psoriasis. A similar gut microbiota profile was present in patients with inflammatory bowel disease [43]. In children with enthesitis-related arthritis (ERA) a different microbiome signature has been observed characterized by (i) less Faecalibacterium prausnitzii and lachnospiraceae family, (ii) a statistically significant increase in Bifidobacterium, and (iii) an abundance of Akkermansia muciniphila [44]. Cluster analysis revealed the presence of two clusters of ERA patients: in cluster one there were high levels of Bacteroides genus; in cluster 2 similar levels of Bacteroides genus, but abundant $A$. muciniphila were observed, indicating Bacteroides and A. muciniphila as associative agents in subsets of ERA patients. The presence of intestinal dysbiosis in AS is suggested by the evidence of over-expression of paneth cells-derived antimicrobial peptides such as human $\alpha$-defensin 5 (HD-5), phospholipase A2 (PLA2), and lysozyme in the ileum of AS patients, with subclinical gut inflammation [26]. This alteration likely represents an important early alteration of the mucosal innate immune component and intestinal host defense in AS. The comparison of the fecal microflora of patients with AS and with that of the controls using molecular methods of analysis demonstrated a higher prevalence of sulfate-reducing bacteria in the feces of the patients [45]. Interestingly, the concentration of serum antibodies reactive with whole cells of Klebsiella pneumoniae or Bacteroides vulgatus was lower in the sera of patients compared with that of controls, and the CD4+ lymphocyte proliferation index, after exposure to homologous Bacteroides cells, was greater in AS than in controls [45]. These findings seem to indicate a possible loss of immunological tolerance to autologous Bacteroides in AS.

Recently, Costello et al. [46] performed culture-independent microbial community profiling, demonstrating differences in the gut microbiome of terminal ileum biopsy specimens between AS patients and healthy controls. Ileal microbial communities of patients with AS differed significantly from that of healthy controls, being characterized by higher abundance of five bacteria families: Lachnospiraceae, Veillonellaceae, Prevotellaceae, Porphyromonadaceae, and Bacteroidaceae. Altogether these findings indicate the presence of different associative bacterial agents in SpA. In this regard, further studies are required to specifically address the role of intestinal dysbiosis in the pathogenesis of $\mathrm{SpA}$ and the influence of host genetics in changing intestinal microbial composition.

Despite a theoretical rationale for probiotic therapy, Jenks $\mathrm{K}$ et al., in a recent randomized controlled trial, did not demonstrate any probiotic benefit in SpA patients [47]. The primary outcome measure of this study was 10\% improvement in the Bath Ankylosing Spondylitis functional index (BASFI). Additional outcome measures were improvements in the Assessments in Ankylosing Spondylitis (ASAS)endorsed core domains: pain, spinal mobility, patient global, peripheral joint, and entheseal scores, stiffness, C-reactive protein, and fatigue. Quality of life and bowel symptoms were quantified using the Ankylosing Spondylitis Quality of Life Questionnaire (ASQoL) and Dudley Inflammatory Bowel Symptom Questionnaire (DISQ). In this study, the probiotic combination administered did not demonstrate significant benefit over the placebo in any of the core domains. 


\section{Dysbiosis in connective tissue diseases}

There is increasing evidence that suggests that the gastrointestinal tract, and in particular, gut dysbiosis could play a potential role also in the pathogenesis and progression of connective tissue diseases (CTDs), such as systemic lupus erythematosus (SLE), Sjögren's syndrome (SS), and systemic sclerosis (SSc).

\section{Systemic lupus erythematosus}

SLE is a chronic multisystem autoimmune disease that can potentially lead to serious organ complications [48]. Changes in intestinal microbiome have been found both in lupus animal models and in SLE patients, however the exact cause-effect relationship between alterations of the gut bacterial composition and the development of the disease remains still to be elucidated. Two different models of interaction between the gut microbiome and the immune system in SLE have been proposed [49]. In the first, it has been hypothesized that, in a pre-clinical stage of SLE, dysbiosis could promote an aberrant mucosal immune response to self-antigens, and as a consequence, enhance a local and systemic inflammatory state with hyperactive antibody production and disease manifestations in predisposed subjects. Conversely, in the other model, dysbiosis could be not a cause, but an epiphenomenon of the altered SLE-related immune reactions toward specific commensal bacterial antigens disturbing intestinal homeostasis with subsequent breakdown of tolerance. Research conducted on animal models has demonstrated both the presence of dysbiosis and immunoregulatory functions of microbiome in lupus-prone mice. Zhang et al. [50] observed that female lupus-prone (Mixed lymphocyte reaction) MRL/lpr mice display an increase in fecal Lachnospiraceae and a decrease in Lactobacillaceae compared to age-matched healthy controls. In addition, a different composition of gut microbiota was observed between the sexes, with an over-representation of Lachnospiraceae in female mice, linked to the development of more severe disease. Remarkably, lupus manifestations were improved with oral retinoid acid treatment concurrently with the Lactobacilli restoration. Analyzing immunologic differences in the gut of female lupus-prone SNF1 mice, which develop a more severe form of nephritis than male mice, Gaudreau et al. [51] demonstrated that female SNF1 mice have more plasma cells and $\alpha 4 \beta 7$ T cells in the Peyer's patches and much higher pro-inflammatory cytokines as IL-6, IL-9, IL-17, IL-22, IFN- $\alpha$, and IFN- $\beta$ in distal ileum than their male counterparts. Although the microbiome composition was not analyzed in this study, it has been hypothesized that the observed mucosal immune differences between the sexes may be the expression of an underlying altered gut bacterial flora. In a recent work, Johnson et al. showed that dietary manipulation may influence gut microbiota composition, and consequently, inflammation and disease progression in lupus SNF1 mice [52]. The authors demonstrated that, compared to SNF1 mice receiving neutral ( $\mathrm{pH}$ 7.0-7.2) water (NW), mice receiving acidified ( $\mathrm{pH} 3.0-3.2)$ water (AW) displayed a delay in nephritis onset, less anti-dsDNA and anti-nucleohistone production, and a reduction in kidney inflammatory infiltrate as well as a reduction in small intestine expression of Th17-related cytokines such as IL-17, IL-21, and IL-23. Gut microbiome analysis revealed significant differences between AW and NW mice with the observation of a promoted growth of Firmicutes bacteria in AW mice, as also previously demonstrated in humans. Moreover, the transfer of gut microbiota by oral gavage from AW to NW mice, interestingly, was able to suppress lupus progression in NW mice. This evidence supports the determining role of intestinal microbiome in promoting development and activity flare of lupus in SNF1 mice. In SLE patients, gut dysbiosis was first observed many years ago by Apperloo-Renkema et al. [53], who demonstrated the presence of different biotypes of Enterobacteriaceae in active SLE than inactive patients and healthy subjects. Recently, Hevia et al. [54] found a lower Firmicutes/Bacteroidetes ratio in SLE patients than in healthy subjects (median ratio 1.97 vs. 4.86; $\mathrm{p}<0.002$ ) and demonstrated that microbiota isolated from SLE patient stool samples was able to promote Th17 differentiation from naïve CD4-positive lymphocytes more than healthy control microbiota. Interestingly, when microbiota was enriched with Treg-inducer bacteria, such as some Clostridia strains, the Th17/Th1 balance was significantly reduced, thus supporting a possible therapeutic approach with probiotics in restoring the imbalance between Th17 and Treg-Th1 present in SLE. Moreover, analyses of fecal microbiota revealed that Firmicutes bacteria, 
which was reduced in SLE patients, positively correlated with serum levels of IFN $\gamma$, a Th1 cytokine that was found slightly reduced in patients, and negatively correlated with IL-17-positive populations in healthy controls [55]. Finally, the authors found a positive correlation between the Synergistetes and the Firmicutes/Bacteroidetes ratio in healthy controls. Synergistetes tended to be reduced when anti-dsDNA titers and IL-6 serum levels were increased and correlated positively with protective natural IgM antibodies against phosphorylcholine [55]. Collectively these findings show that both lymphocytic polarizations and natural protective immune defenses could be influenced by the gut microbiota composition in SLE patients. Metabolomic studies [56] were also performed in order to investigate the presence of potential disease-related alterations of fecal metabolites in SLE patients. A deficiency in amino acid metabolism and in metabolites associated with pyrimidine and purine was observed in the stool of patients than healthy controls, but with differences too limited to establish what could be its real pathophysiologic implication.

\section{Sjogren's syndrome}

Sjogren's syndrome (SS) is a systemic chronic inflammatory disorder characterized by lymphocytic infiltrates in exocrine organs [57]. The majority of studies about dysbiosis in SS have evaluated the implications of hyposalivation on the oral bacterial flora alterations [58,59]. According to the molecular mimicry model, SS would be the result of a still unknown complex network of interactions between genetic and environmental factors, likely infectious-related antigens, that could trigger an abnormal self-perpetuating pro-inflammatory state, leading to autoimmunity and lymphoproliferation [57]. While the potential role of viruses in SS pathogenesis has been widely explored, only one study that focused on a possible implication of bacteria in SS development is currently available. To test this hypothesis, Szymula et al. [60] created Ro62/SSA reactive T cell hybridomas from HLA-DR3 transgenic mice for the susceptibility for SS and SLE, and evaluated their reactivity against oral, gut, and skin bacteria-derived peptides. The authors found that several antigens from normal commensal microbiota were able to activate Ro62/SSA T cell hybridomas. The most potent stimulator was identified in the von Willebrand factor type A domain protein (vWFA), mainly expressed by Bacteroides intestinalis in the gut and Capnocytophaga ochracea in the oral mucosa. These findings suggest that the activation of $\mathrm{T}$ cell in SS could be primed at the mucosal level by exposure to mimicry antigens from normal commensal bacterial flora, contributing to the initiation of autoimmune responses in SS.

\section{Systemic sclerosis}

Research studies examining the role of dysbiosis in Systemic sclerosis (SSc) are lacking. A recent study [61] was undertaken to investigate the hypothesis that SSc could be associated with altered gut bacterial composition in the colon tract, and mainly that gastrointestinal (GI) symptoms in SSc patients could mirror some underlying microbiome alterations. The authors demonstrated that there were significant differences in the microbial communities found in mucosal lavage samples from the cecum and sigmoid tracts of SSc patients when compared with healthy controls. SSc patients showed significantly decreased levels of commensal flora, such as Faecalibacterium and Clostridium, and increased levels of Fusobacterium and $\gamma$-Proteobacteria, compared to healthy subjects. Bifidobacterium and Lactobacillus were also increased in patients with SSc. Moreover, SSc patients with gastrointestinal symptoms had an abundance of Fusobacterium and a decrease in the levels of Bacteroides fragilis compared to patients without or with mild symptoms. Thus, this study demonstrated a particular bacterial signature in SSc patients that may perpetuate immunologic alterations and contribute to the clinical manifestations in SSc.

\section{Summary}

Overall, available evidence confirms the existence of a strong association between intestinal dysbiosis and RA and connective tissue diseases. Further studies are however needed to establish the clinical relevance of intestinal inflammation in these patients and the exact impact of gut microbiota in influencing the initiation of autoimmune response and the modulation of the activity in these diseases. Subclinical gut inflammation and a relevant dysbiosis is present in a significant percentage of AS 
patients, and it seems to represent the site where close interaction occurs between the host microbiota and the immune system. The identification of a specific "dysbiotic signature" for each disease could allow the development of a complimentary therapeutic approach based on treatments targeted to restore a favorable microbiota balance.

\section{Practice points}

- The gastrointestinal tract plays a key role in the pathogenesis of SpA by modulating innate and adaptive immune responses.

- Dysbiosis seems to be relevant in modulating intestinal inflammation in SpA.

- Dysbiosis occurs in patients with CTD and RA.

\section{Research agenda}

- We need to better study the role of gastrointestinal tract in the pathogenesis of RA and CTD. In particular, we have to clarify whether a "subclinical gut inflammation" occurs in these patients and whether it may modulate local and systemic responses.

\section{Disclosures}

The authors declare no competing interests.

\section{References}

[1] Peterson LW, Artis D. Intestinal epithelial cells: regulators of barrier function and immune homeostasis. Nat Rev Immunol 2014; $14: 141-53$.

[2] Kåhrström CT, Pariente N, Weiss U. Intestinal microbiota in health and disease. Nature 2016;535:47. http://dx.doi.org/10. 1038/535047a.

[3] Smolen JS, Aletaha D, McInnes IB. Rheumatoid arthritis. Lancet 2016;388:2023-38. http://dx.doi.org/10.1016/S01406736(16)30173-8. pii: S0140-6736(16)30173-8.

[4] McInnes IB, Schett G. The pathogenesis of rheumatoid arthritis. N Engl J Med 2011;365(23):2205-19.

[5] Jenkins RT, Rooney PJ, Jones DB, et al. Increased intestinal permeability in patients with rheumatoid arthritis: a side-effect of oral nonsteroidal anti-inflammatory drug therapy? Br J Rheumatol 1987;26(2):103-7.

[6] Segal AW, Isenberg DA, Hajirousou V, et al. Preliminary evidence for gut involvement in the pathogenesis of rheumatoid arthritis? Br J Rheumatol 1986;25(2):162-6.

[7] Bjarnason I, Williams P, So A, et al. Intestinal permeability and inflammation in rheumatoid arthritis: effects of nonsteroidal anti-inflammatory drugs. Lancet 1984;2(8413):1171-4.

[8] Wegner N, Wait R, Sroka A, et al. Peptidylarginine deiminase from Porphyromonas gingivalis citrullinates human fibrinogen and $\alpha$-enolase: implications for autoimmunity in rheumatoid arthritis. Arthritis Rheum 2010;62(9):2662-72.

[9] Griffen AL, Becker MR, Lyons SR, et al. Prevalence of Porphyromonas gingivalis and periodontal health status. J Clin Microbiol 1998;36(11):3239-42.

[10] Liu X, Zeng B, Zhang J, et al. Role of the gut microbiome in modulating arthritis progression in mice. Sci Rep 2016;6: 30594. http://dx.doi.org/10.1038/srep30594.

[11] Block KE, Zheng Z, Dent AL, et al. Gut microbiota regulates K/BxN autoimmune arthritis through follicular helper T but not Th17 cells. J Immunol 2016;196(4):1550-7.

[12] Johnson S, Sidebottom D, Bruckner F, et al. Identification of Mycoplasma fermentans in synovial fluid samples from arthritis patients with inflammatory disease. J Clin Microbiol 2000;38(1):90-3.

[13] Newkirk MM, Zbar A, Baron M, et al. Distinct bacterial colonization patterns of Escherichia coli subtypes associate with rheumatoid factor status in early inflammatory arthritis. Rheumatology 2010;49(7):1311-6.

[14] Senior BW, Anderson GA, Morley KD, et al. Evidence that patients with rheumatoid arthritis have asymptomatic 'nonsignificant' Proteus mirabilis bacteriuria more frequently than healthy controls. J Infect 1999;38(2):99-106.

[15] Sandberg ME, Bengtsson C, Klareskog L, et al. Recent infections are associated with decreased risk of rheumatoid arthritis: a population-based case-control study. Ann Rheum Dis 2015;74(5):904-7.

[16] Chen J, Wright K, Davis JM, et al. An expansion of rare lineage intestinal microbes characterizes rheumatoid arthritis. Genome Med 2016;8(1):43. 
[17] Liu X, Zou Q Zeng B, et al. Analysis of fecal Lactobacillus community structure in patients with early rheumatoid arthritis. Curr Microbiol 2013;67(2):170-6.

*[18] Zhang X, Zhang D, Jia H, et al. The oral and gut microbiomes are perturbed in rheumatoid arthritis and partly normalized after treatment. Nat Med 2015;21(8):895-905.

[19] Vaahtovuo J, Munukka E, Korkeamäki M, et al. Fecal microbiota in early rheumatoid arthritis. J Rheumatol 2008;35(8): $1500-5$.

[20] Vaghef-Mehrabany E, Alipour B, Homayouni-Rad A, et al. Probiotic supplementation improves inflammatory status in patients with rheumatoid arthritis. Nutrition 2014 Apr;30(4):430-5.

[21] Taurog JD, Chhabra A, Colbert RA. Ankylosing spondylitis and axial spondyloarthritis. N Engl J Med 2016;374(26): 2563-74.

*[22] Mielants H, Veys EM, Cuvelier C, et al. Ileocolonoscopic findings in seronegative spondylarthropathies. Br J Rheumatol 1988;27(Suppl. 2):95-105.

[23] Van Praet L, Jans L, Carron P, et al. Degree of bone marrow oedema in sacroiliac joints of patients with axial spondyloarthritis is linked to gut inflammation and male sex: results from the GIANT cohort. Ann Rheum Dis 2014;73(6):1186-9.

[24] Mielants H, Veys EM, De Vos M, et al. The evolution of spondyloarthropathies in relation to gut histology. I. Clinical aspects. J Rheumatol 1995;22(12):2266-72.

[25] Ciccia F, Accardo-Palumbo A, Alessandro R, et al. Interleukin-22 and interleukin-22-producing NKp44+ natural killer cells in subclinical gut inflammation in ankylosing spondylitis. Arthritis Rheum 2012;64(6):1869-78.

*[26] Ciccia F, Bombardieri M, Rizzo A, et al. Over-expression of cell-derived anti-microbial peptides in the gut of patients with ankylosing spondylitis and subclinical intestinal inflammation. Rheumatol Oxf 2010;49(11):2076-83.

*[27] Ciccia F, Guggino G, Ferrante A, et al. Interleukin-9 overexpression and Th9 polarization characterize the inflamed gut, the synovial tissue, and the peripheral blood of patients with psoriatic arthritis. Arthritis Rheumatol 2016;68(8):1922-31.

[28] Ciccia F, Lucchetti MM, Guggino G, et al. Gut dysbiosis in patients with HLA-B27+ Ankylosing Spondylitis is associated with ileitis, down-regulation of tight junction proteins, increased serum levels of LPS and monocytes anergy. Ann Rheum Dis 2015;74(Suppl. 2):148.

[29] YR1 Mahida, Makh S, Hyde S, et al. Effect of Clostridium difficile toxin A on human intestinal epithelial cells: induction of interleukin 8 production and apoptosis after cell detachment. Gut 1996;38(3):337-47.

*[30] Cypers H, Varkas G, Beeckman S, et al. Elevated calprotectin levels reveal bowel inflammation in spondyloarthritis. Ann Rheum Dis 2016 Jul;75(7):1357-62.

[31] Sands BE. Biomarkers of inflammation in inflammatory bowel disease. Gastroenterology 2015;149(5):1275-85.

[32] Ciccia F, Ferrante A, Triolo G. Intestinal dysbiosis and innate immune responses in axial spondyloarthritis. Curr Opin Rheumatol 2016;28(4):352-8.

[33] Ciccia F, Bombardieri M, Principato A, et al. Overexpression of interleukin-23, but not interleukin-17, as an immunologic signature of subclinical intestinal inflammation in ankylosing spondylitis. Arthritis Rheum 2009;60(4):955-65.

[34] Ciccia F, Accardo-Palumbo A, Giardina A, et al. Expansion of intestinal CD4 + CD25(high) Treg cells in patients with ankylosing spondylitis: a putative role for interleukin 10 in preventing Th17 response. Arthritis Rheum 2010;62(12): 3625-34.

[35] Newberry RD, Lorenz RG. Organizing a mucosal defense. Immunol Rev 2005 Aug;206:6-21.

[36] Eberl G. Inducible lymphoid tissues in the adult gut: recapitulation of a fetal developmental pathway? Nat Rev Immunol 2005;5:413-20.

*[37] Ciccia F, Guggino G, Rizzo A, et al. Type 3 innate lymphoid cells producing IL-17 and IL-22 are expanded in the gut, in the peripheral blood, synovial fluid and bone marrow of patients with ankylosing spondylitis. Ann Rheum Dis 2015;74:1739-47.

[38] Artis D, Spits H. The biology of innate lymphoid cells. Nature 2015;517:293-301.

[39] Ciccia F, Rizzo A, Guggino G, et al. Clinical efficacy of a4 integrin block with natalizumab in Ankylosing Spondylitis. Ann Rheum Dis 2016;75:2053-4. annrheumdis-2016-209748.

[40] Franciszkiewicz K, Salou M, Legoux F, et al. MHC class-I-related molecule, MR1, and mucosal associated invariant T cells. Immunol Rev 2016:272(1):120-38.

*[41] Gracey E, Qaiyum Z, Almaglouth I, et al. IL-7 primes IL-17 in mucosal-associated invariant T (MAIT) cells, which contribute to the Th17-axis in ankylosing spondylitis. Ann Rheum Dis 2016 May 10. annrheumdis-2015-208902.

[42] Tito RY, Cypers H, Joossens M, et al. Dialister as microbial marker of disease activity in spondyloarthritis. Arthritis Rheumatol 2016 Jul 7. http://dx.doi.org/10.1002/art.39802.

*[43] Scher JU, Ubeda C, Artacho A, et al. Decreased bacterial diversity characterizes the altered gut microbiota in patients with psoriatic arthritis, resembling dysbiosis in inflammatory bowel disease. Arthritis Rheumatol 2015 Jan;67(1):128-39.

[44] Stoll ML, Kumar R, Morrow CD, et al. Altered microbiota associated with abnormal humoral immune responses to commensal organisms in enthesitis-related arthritis. Arthritis Res Ther 2014;16(6):486.

[45] Stebbings S, Munro K, Simon MA, et al. Comparison of the faecal microflora of patients with ankylosing spondylitis and controls using molecular methods of analysis. Rheumatol Oxf 2002:41(12):1395-401.

*[46] Costello ME, Ciccia F, Willner D, et al. Intestinal dysbiosis in ankylosing spondylitis. Arthritis Rheumatol 2015;3:686-91.

[47] Jenks K, Stebbings S, Burton J, et al. Probiotic therapy for the treatment of spondyloarthritis: a randomized controlled trial. J Rheumatol 2010;37(10):2118-25.

[48] Carter EE, Barr SG, Clarke AE. The global burden of SLE: prevalence, health disparities and socioeconomic impact. Nat Rev Rheumatol 2016 Aug 25;12:605-20. http://dx.doi.org/10.1038/nrrheum.2016.137.

[49] López P, Sánchez B, Margolles A, et al. Intestinal dysbiosis in systemic lupus erythematosus: cause or consequence? Curr Opin Rheumatol 2016 Sep;28(5):515-22.

[50] Zhang H, Liao X, Sparks JB, et al. Dynamics of gut microbiota in autoimmune lupus. Appl Environ Microbiol 2014 Dec 80(24):7551-60.

[51] Gaudreau MC, Johnson BM, Gudi R, et al. Gender bias in lupus: does immune response initiated in the gut mucosa have a role? Clin Exp Immunol 2015 Jun;180(3):393-407.

[52] Johnson BM, Gaudreau MC, Al-Gadban MM, et al. Impact of dietary deviation on disease progression and gut microbiome composition in lupus-prone SNF1 mice. Clin Exp Immunol 2015 Aug;181(2):323-37. 
[53] Apperloo-Renkema HZ, Bootsma H, Mulder BI, et al. Host-microflora interaction in systemic lupus erythematosus (SLE): colonization resistance of the indigenous bacteria of the intestinal tract. Epidemiol Infect 1994 Apr;112(2):367-73.

[54] Hevia A, Milani C, López P, et al. Intestinal dysbiosis associated with systemic lupus erythematosus. MBio 2014 Sep 30 ; 5(5). e01548-14.

*[55] López P, de Paz B, Rodríguez-Carrio J, et al. Th17 responses and natural IgM antibodies are related to gut microbiota composition in systemic lupus erythematosus patients. Sci Rep 2016 Apr 5;6:24072.

[56] Rojo D, Hevia A, Bargiela R, et al. Ranking the impact of human health disorders on gut metabolism: systemic lupus erythematosus and obesity as study cases. Sci Rep 2015 Feb 6;5:8310.

[57] Mavragani CP, Moutsopoulos HM. Sjogren's syndrome. Ann Rev Pathol 2014;9:273-85.

[58] Leung KC, Leung WK, McMillan AS. Supra-gingival microbiota in Sjögren's syndrome. Clin Oral Investig 2007 Dec;11(4): $415-23$.

[59] Shinozaki S, Moriyama M, Hayashida JN, et al. Close association between oral Candida species and oral mucosal disorders in patients with xerostomia. Oral Dis 2012 Oct;18(7):667-72.

[60] Szymula A, Rosenthal J, Szczerba BM, et al. T cell epitope mimicry between Sjögren's syndrome Antigen A (SSA)/Ro60 and oral, gut, skin and vaginal bacteria. Clin Immunol 2014 May-Jun;152(1-2):1-9.

*[61] Volkmann ER, Chang YL, Barroso N, et al. Association of systemic sclerosis with a unique colonic microbial consortium. Arthritis Rheumatol 2016 Jun;68(6):1483-92. 